\title{
Rigorous Method of Weights Calculation in Adjustment of Measurement Data with Different Qualities
}

Ye Xiaoming ( $\nabla$ xmye@sgg.whu.edu.cn )

Wuhan University https://orcid.org/0000-0002-8227-4181

Ding Shijun

Wuhan University

Liu Haibo

China Earthquake Administration

\section{Research Article}

Keywords: measurement theory, random variable, uncertainty, survey adjustment, weight

Posted Date: August 19th, 2021

DOI: https://doi.org/10.21203/rs.3.rs-146290/v4

License: (c) (i) This work is licensed under a Creative Commons Attribution 4.0 International License.

Read Full License 


\title{
Rigorous Method of Weights Calculation in Adjustment of Measurement Data with Different Qualities
}

\author{
Ye Xiaoming ${ }^{1 *}$ Ding shijun ${ }^{1}$ Liu Haibo ${ }^{23}$ \\ 1. School of Geodesy and Geomatics, Wuhan University, Wuhan, Hubei, China, 430079. \\ 2. Institute of Seismology, China Earthquake Administration, Wuhan, Hubei 430071, China. \\ 3. Wuhan Institute of Earthquake Metrological Verification and Measurement Engineering, \\ Wuhan, Hubei 430071, China
}

\begin{abstract}
In the traditional measurement theory, precision is defined as the dispersion of measured value, and is used as the basis of weights calculation in the adjustment of measurement data with different qualities, which leads to the trouble that trueness is completely ignored in the weight allocation. In this paper, following the pure concepts of probability theory, the measured value (observed value) is regarded as a constant, the error as a random variable, and the variance is the dispersion of all possible values of an unknown error. Thus, a rigorous formula for weights calculation and variance propagation is derived, which solves the theoretical trouble of determining the weight values in the adjustment of multi-channel observation data with different qualities. The results show that the optimal weights are not only determined by the covariance array of observation errors, but also related to the model of adjustment.
\end{abstract}

Keywords: measurement theory, random variable, uncertainty, survey adjustment, weight.

\section{Introduction}

The traditional measurement error theory is interpreted following the logic of error classification, in which the measured value (observed value) and random error are regarded as random variables with variance, while the systematic error is regarded as constant without variance $^{[1,2,3,4,5,6]}$. In this way, when multiple observations with different qualities are obtained from a single measurand, according to this conceptual logic, the weight of each observed value is calculated by its precision, and the final measured value is equal to the weighted average value of all observed values.

However, the biggest trouble faced by this method is that the systematic error of each observation is different from each other. Some observations with large systematic error but small random error get larger weights, which can make the final measured value have better precision, but its trueness will be worse. Although we can explain the existing theory based on the assumption that the systematic error does not exist or can be ignored, this assumption is usually not tenable in actual measurement.

For example, in the field of geodesy, the multiplicative constant error of a geodimeter is considered as systematic error, its measured value given by metrology field is usually used to correct the observed value, and people think that the residual systematic error after correction can be ignored. However, in the field of instrument manufacturing, the multiplicative constant error of geodimeter is originally the residual error after correction, which comes from the residual error of quartz crystal frequency after temperature correction, and still varies with temperature. In the field of instrument, the limited range of this error is also given. Therefore, it is actually meaningless to use a test sample given by metrology field to correct the observed value.

Therefore, this concept of systematic error often makes the measurement practice in trouble.

For example, in 2020, the National Bureau of surveying, mapping and geographic information of China announced that the elevation of Mount Everest is $8848.86 \mathrm{~m}$, which is actually an

\footnotetext{
*xmye@sgg.whu.edu.cn
} 
adjustment value obtained from the trigonometric leveling data of geodimeter and GNSS satellite survey data according to a certain weight proportion. However, at present, we have not seen the public information about weight calculation, nor the official data about error evaluation.

In recent years, literature ${ }^{[7,8,9,10,11,12,13]}$ proposed an interpretation method of measurement theory based new concepts, which clearly pointed out that the traditional measurement theory's understanding of constant and random variable concepts is inconsistent with probability theory. Because the constant in probability theory refers to a numerical value, and the random variable refers to a quantity whose numerical value is unknown, this theory regards the observed value (measured value) as a constant, the error and the true value as random variables, and the variance of error is the dispersion of all possible values of the error. In this conceptual system, errors cannot be classified as systematic error and random error.

Although these documents have been published formally, they do not deal with the problem of weight calculation in multi-channel data adjustment, so this paper will complete the mathematical deduction of this calculation principle.

\section{Basic concepts}

\subsection{Constant and random variable}

In probability theory, a numerical value is regarded as a constant, but a random variable is an unknown quantity without numerical value. To study the random variable, people can only use all its possible values (sample space) to describe its probability interval.

\subsection{Mathematical expectation and variance}

For a random variable $X$ with sample space $\left\{x_{i}\right\}$, there is $X \in\left\{x_{i}\right\}, p_{i}$ is the probability of occurrence of event $\left\{X=x_{i}\right\}$, that is, $p_{i}=P\left\{X=x_{i}\right\}$ (the probability density function $p(x)$ is used to express continuous random variable), and its mathematical expectation and variance are respectively defined as:

$$
\left\{\begin{array}{l}
E(X)=\sum_{i=1}^{n} p_{i} x_{i} \text { or } \quad E(X)=\int_{-\infty}^{+\infty} x p(x) d x \\
\sigma^{2}(X)=E[X-E(X)]^{2}
\end{array}\right.
$$

Its meaning is that all possible values of random variable $X$ constitute a density distribution interval (Frequency school) with $E(X)$ as the center and $\sigma^{2}(X)$ as the width evaluation, or the random variable $X$ exists within a probability interval (Bayesian school) described by $E(X)$ and $\sigma^{2}(X)$. In other words, the mathematical expectation $E(X)$ and the variance $\sigma^{2}(X)$ are the evaluation of the probability interval of $X$.

Because all possible values of a constant $C$ are itself, there are:

$$
\left\{\begin{array}{l}
E(C)=C \\
\sigma^{2}(C)=E[C-E(C)]^{2}=0
\end{array}\right.
$$

For example, when there is $x=8848.86$, there must be $E(x)=E(8848.86)=8848.86$ and $\sigma^{2}(x)=\sigma^{2}(8848.86)=0$.

\subsection{The origin of the conceptual trouble in traditional measurement theory}

In Section 4.2 of literature ${ }^{[2]}$ and Section 5.17 of literature ${ }^{[3]}$, a method process is described as follows:

When obtaining $n$ observed values $q_{k}$, the final measured value $\bar{q}$ is

$$
\bar{q}=\frac{1}{n} \sum_{k=1}^{n} q_{k}
$$


The variance of each observed value $q_{k}$ is

$$
\sigma^{2}\left(q_{k}\right) \approx \frac{1}{n-1} \sum_{j=1}^{n}\left(q_{j}-\bar{q}\right)^{2}
$$

The variance of the final measured value $q$ is

$$
\sigma^{2}(\bar{q})=\frac{\sigma^{2}\left(q_{k}\right)}{n}
$$

Obviously, in formula (2-3), $\bar{q}$ and each $q_{k}$ respectively represent a numerical value; but in formulas (2-4) and (2-5), these numerical values $\bar{q}$ and $q_{k}$ are given a variance that is not zero, which obviously violates the formula (2-2).

For example, a DC voltage is repeatedly measured 100 times, and there are 50 observed values of $5.0 \mathrm{~V}$ and 50 observed values of $5.1 \mathrm{~V}$. According to formula (2-3) (2-4) (2-5), we can get:

$$
\begin{gathered}
\bar{q}=(50 \times 5.0+50 \times 5.1) / 100=5.05(V) \\
\sigma^{2}(5.0)=\sigma^{2}(5.1) \approx 0.0025\left(V^{2}\right) \\
\sigma^{2}(\bar{q})=\sigma^{2}(5.05)=0.000025\left(V^{2}\right) .
\end{gathered}
$$

That is, in formula (2-1), all numerical values $x_{i}$ or $x$ are used to describe variable $X$, but the formulas (2-4) and (2-5) do not follow this principle and imposes the concept of variable on numerical values itself.

Because the numerical values (observed value and measured value) are regarded as random variable, the traditional measurement theory uses Fig 1 to interpret its basic measurement concepts, which further leads to the philosophy of error classification and misleads the conceptual difference between trueness and precision. Formulas (2-4) and (2-5) are the mathematical expressions of the so-called precision concept.

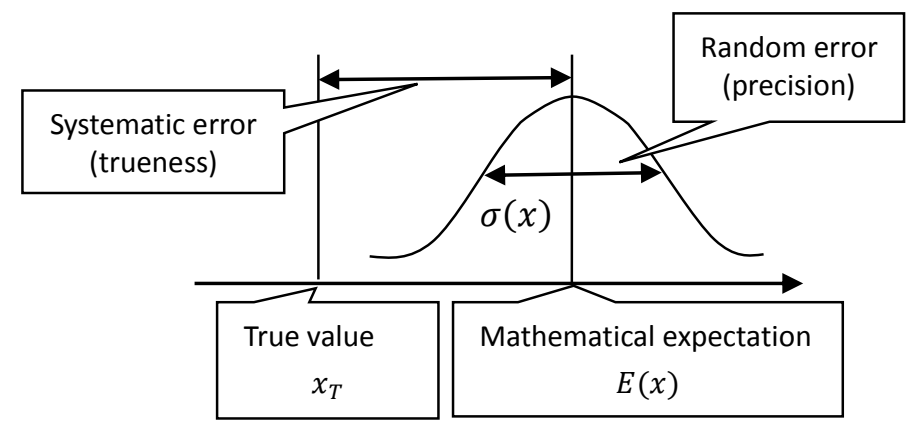

Fig 1. Schematic diagram in traditional measurement theory

In a word, the concept of error classification in traditional measurement theory is obtained by misinterpreting the concept of random variable, so the classification concept of precision and trueness is actually not correct ${ }^{[7][8][9][10][11][12][13]}$.

\subsection{The probability expressions of measured value, error and true value}

In references ${ }^{[7,8,9,10,11,12,13]}$, Fig 2 is used to interpret its basic measurement concepts, which is different from the traditional measurement theory. Among them, the final measured value is an observed value $x_{0}$ which is a sample within all possible observed values $\left\{x_{i}\right\}$, and $\left\{x_{i}\right\}$ is the sample space of random variable $X$. 


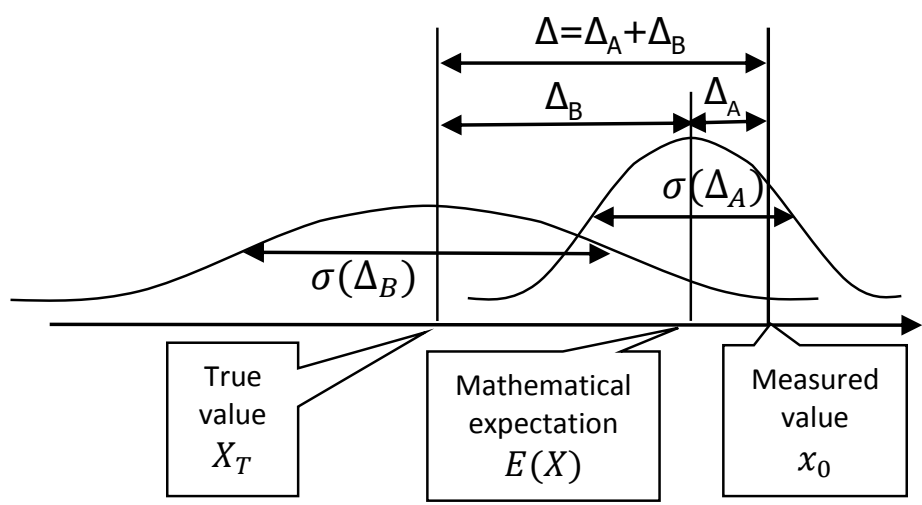

Fig 2. Conceptual sketch of new concept theory

Because $x_{0}$ is a numerical value, according to the formula (2-2), there must be:

$$
\left\{\begin{array}{l}
E\left(x_{0}\right)=x_{0} \\
\sigma^{2}\left(x_{0}\right)=0
\end{array}\right.
$$

For the unknown deviation $\Delta_{A}=x_{0}-E(X)$, because it has the same sample space $\left\{x_{i}-E(X)\right\}$ as the random variable $\Delta X=X-E(X)$, we can use $\Delta X=X-E(X)$ to express the deviation $\Delta_{A}$, that is, $\Delta_{A}=X-E(X)$. Therefore, there are

$$
\left\{\begin{array}{l}
E\left(\Delta_{A}\right)=E[X-E(X)]=0 \\
\sigma^{2}\left(\Delta_{A}\right)=E\left(\Delta_{A}{ }^{2}\right)=E[X-E(X)]^{2}
\end{array}\right.
$$

Its concept meaning is that variance $\sigma^{2}\left(\Delta_{A}\right)$ is the dispersion (Frequency School) of all possible values $\left\{x_{i}-E(X)\right\}$ of deviation $\Delta_{A}$, and is also the evaluation value of the probability interval (Bayesian School) of deviation $\Delta_{A}$.

For the unknown deviation $\Delta_{B}=E(X)-X_{T}$, because it comes from the previous measurement, its formation principle is similar to that of deviation $\Delta_{A}$, and it also has all its possible values, so there are

$$
\left\{\begin{array}{l}
E\left(\Delta_{B}\right)=0 \\
\sigma^{2}\left(\Delta_{B}\right)=E\left(\Delta_{B}{ }^{2}\right)
\end{array}\right.
$$

For example, the field of geodesy generally considers that the multiplicative constant error of geodimeter is a systematic error without variance, but from the perspective of instrument manufacturing, this error is the random error, and the instrument manufacturer also uses all its possible values to count its probability interval (usually expressed by the maximum permissible error MPE). That is, the multiplicative constant error of geodimeter also has its variance like the output deviation in the field of geodesy.

That is to say, both $\Delta_{A}$ and $\Delta_{B}$ are unknown deviations, have their own variances, and cannot be classified as random error and systematic error respectively. That is, the concepts of precision and trueness were abolished.

In this way, there are

$$
\begin{gathered}
\Delta=\Delta_{A}+\Delta_{B} \\
\begin{aligned}
E(\Delta) & =E\left(\Delta_{A}\right)+E\left(\Delta_{B}\right) \\
& =0
\end{aligned}
\end{gathered}
$$




$$
\begin{aligned}
\sigma^{2}(\Delta) & =E[\Delta-E(\Delta)]^{2} \\
& =E\left(\Delta^{2}\right) \\
& =E\left(\Delta_{A}+\Delta_{B}\right)^{2} \\
& =\sigma^{2}\left(\Delta_{A}\right)+\sigma^{2}\left(\Delta_{B}\right)
\end{aligned}
$$

The variance $\sigma^{2}(\Delta)$ is the evaluation value of the probability range (Bayesian School) of the total error $\Delta$, is also the dispersion (Frequency School) of all possible values of the total error $\Delta$, and is called as the uncertainty of the error $\Delta$. Please note that this uncertainty is not the dispersion of the measured value interpreted by the traditional measurement theory.

Therefore, for the true value $X_{T}$, there are:

$$
\begin{aligned}
X_{T} & =x_{0}-\Delta \\
E\left(X_{T}\right) & =E\left(x_{0}\right)-E(\Delta) \\
& =x_{0} \\
\sigma^{2}\left(X_{T}\right) & =E\left[X_{T}-E\left(X_{T}\right)\right]^{2} \\
& =E\left(x_{0}-\Delta-x_{0}\right)^{2} \\
& =\sigma^{2}(\Delta)
\end{aligned}
$$

In conclusion, the above concepts are summarized in Table $2^{[10][11][12]}$.

Table 2. The probability expressions of measured value, error and true value

\begin{tabular}{|c|c|c|}
\hline Measured value $x_{0}$ & Error $\Delta$ & True value $X_{T}$ \\
\hline$\left\{\begin{array}{l}E\left(x_{0}\right)=x_{0} \\
\sigma^{2}\left(x_{0}\right)=0\end{array}\right.$ & $\left\{\begin{array}{l}E(\Delta)=0 \\
\sigma^{2}(\Delta)=E\left(\Delta^{2}\right)\end{array}\right.$ & $\left\{\begin{array}{l}E\left(X_{T}\right)=x_{0} \\
\sigma^{2}\left(X_{T}\right)=\sigma^{2}(\Delta)\end{array}\right.$ \\
\hline
\end{tabular}

The above conclusion is derived by using an observed value $x_{0}$ as the final measured value. When obtaining multiple observations $x_{i}$, the submission method of the best measured value is shown in Section 3.

\subsection{Regularity and randomness of error}

Any error has variance to evaluate its probability range, and the regular error is of course the same, because the variance of error is the dispersion of all its possible values. Fig 3 is a conceptual diagram describing the regularity and randomness of the periodic error of the geodimeter. When observing the corresponding relationship between the error value and the range, we can see the regularity. When observing the density distribution of the error values, we can see the randomness.

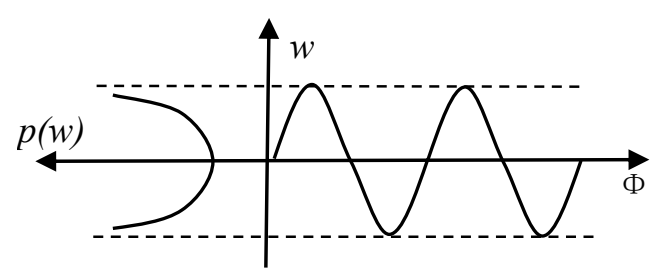

Fig 3. Regularity and randomness of sine error

\subsection{The contribution form of error to repeated measurement}

Because errors vary with various measurement conditions, the variation of measurement conditions in repeated measurement determines the contribution form of errors to repeated observations (deviation, dispersion, outlier or both). It is precisely because errors can lead to the dispersion of repeated observations, so the variance of any error can be obtained through experimental statistics, and these data exist in the instrument specifications or instructions.

\subsection{Contribution form of error to final measured value}


The contribution form of error to the final measured value is only to make it deviate from the true value. This is because, after the measurement adjustment, the final measured value is unique, and the difference between it and the true value is a unique unknown deviation.

\subsection{The law of covariance propagation}

Because every error has its variance, the concept of variance can be extended to any two errors, which is called covariance. It comes from the common component of the two errors.

$$
\sigma\left(\Delta x_{j} \Delta x_{k}\right)=E\left(\Delta x_{j} \Delta x_{k}\right)
$$

Thus, for an error array $\mathbf{\Delta} \mathbf{X}=\left(\Delta x_{1}, \Delta x_{2}, \cdots, \Delta x_{n}\right)^{\mathrm{T}}$, the definition of variance is as follows:

$$
\mathbf{D}(\boldsymbol{\Delta} \mathbf{X})=E\left(\boldsymbol{\Delta} \mathbf{X} \boldsymbol{\Delta} \mathbf{X}^{\mathrm{T}}\right)=\left[\begin{array}{cccc}
\sigma_{11}^{2} & \sigma_{12} & \cdots & \sigma_{1 \mathrm{n}} \\
\sigma_{21} & \sigma_{22}^{2} & \cdots & \sigma_{2 \mathrm{n}} \\
\vdots & \vdots & & \vdots \\
\sigma_{\mathrm{n} 1} & \sigma_{\mathrm{n} 2} & \cdots & \sigma_{\mathrm{nn}}^{2}
\end{array}\right]
$$

Therefore, according to the definition of variance (2-16), when there is an error propagation equation $\Delta \mathbf{Z}=\mathbf{K} \cdot \Delta \mathbf{X}$, we can get:

$$
\mathbf{D}(\Delta \mathbf{Z})=\mathbf{K} \cdot \mathbf{D}(\Delta \mathbf{X}) \cdot \mathbf{K}^{\mathrm{T}}
$$

This is the law of covariance propagation, which is the propagation relation of probability interval between errors, or the propagation relation of the dispersion of all possible values of errors.

After the data processing, the difference between the measured value and the true value is an unknown deviation. Therefore, the task of measurement is to adjust the measured value to minimize the variance of the deviation and submit its value.

\section{The solution of weight values}

The smaller the error is, the better the quality of the measured value is. However, we don't know the error value, and can only use the variance of the error to describe its probability interval, so we can only think that the quality of the measured value is the best when the variance of the error is the smallest. Therefore, the mathematical problem we are faced with is to minimize the variance of the error of the final measured value by assigning the weights of each observed value.

\subsection{Direct measurement model for single measurand}

As shown in Fig 2, when $n$ different observed values $x_{i}$ are obtained by repeated observation of a measurand, assuming that the true value is $Y$, the error equation is as follows:

$$
\left[\begin{array}{c}
V_{1} \\
V_{2} \\
\vdots \\
V_{n}
\end{array}\right]=\left[\begin{array}{c}
x_{1} \\
x_{2} \\
\vdots \\
x_{n}
\end{array}\right]-\left[\begin{array}{c}
1 \\
1 \\
\vdots \\
1
\end{array}\right][Y]
$$

The error sample set $\left\{V_{1}, V_{2}, \cdots, V_{n}, \cdots\right\}$ constitutes a sample space of error $V$. Therefore, according to the formula in Table 2, there are:

$$
\begin{aligned}
\sigma^{2}(V) & =E\left(V^{2}\right) \\
& \approx \frac{\sum V_{i}^{2}}{n} \\
& =\frac{1}{n}\left(x_{1}^{2}+x_{2}^{2}+\cdots+x_{n}^{2}-2 x_{1} Y-2 x_{2} Y-\cdots-2 x_{n} Y+n Y^{2}\right)
\end{aligned}
$$


The best measured value $y$ is the value when $\sigma^{2}(V)$ or $\sum V_{i}^{2}$ takes the minimum value, and this is a minimum problem of quadratic function, so making $\left.\frac{d\left(\sigma^{2}(V)\right)}{d Y}\right|_{Y=y}=0$, the best measured value is obtained as follows:

$$
y=\frac{1}{n} \sum_{i=1}^{n} x_{i}
$$

This is the least square method. As you can see, in the above deduction process, each $x_{i}$ and $y$ do not represent a random variable, which is consistent with the conceptual principle in Section 2 .

Of course, formula (3-3) is actually an equal weight processing method, that is, the weight of each observation $x_{i}$ is $\frac{1}{n}$. However, if $n$ observation errors $\Delta x_{i}$ have different variances and covariances, that is, the quality of each observation $x_{i}$ is different from each other, then the equal weight processing is not scientific. At this time, different weights must be given to each observed value to ensure that the quality of the final measured value is the best. Therefore, in order to study each weight $p_{i}$ when $\sum P_{i} V_{i}^{2}$ takes the minimum value, the equation (3-1) is modified as:

$$
\left[\begin{array}{c}
P_{1}^{1 / 2} V_{1} \\
P_{2}^{1 / 2} V_{2} \\
\vdots \\
P_{n}^{1 / 2} V_{n}
\end{array}\right]=\left[\begin{array}{c}
P_{1}^{1 / 2} x_{1} \\
P_{2}^{1 / 2} x_{2} \\
\vdots \\
P_{n}^{1 / 2} x_{n}
\end{array}\right]-\left[\begin{array}{cccc}
P_{1}^{1 / 2} & 0 & \cdots & 0 \\
0 & P_{2}^{1 / 2} & \cdots & 0 \\
\vdots & \vdots & \vdots & \vdots \\
0 & 0 & \cdots & P_{n}^{1 / 2}
\end{array}\right]\left[\begin{array}{c}
1 \\
1 \\
\vdots \\
1
\end{array}\right][Y]
$$

Similarly, by using the least square method, there is

$$
y=\left(P_{1} x_{1}+P_{2} x_{2}+\cdots+P_{n} x_{n}\right) / \sum_{i=1}^{n} P_{i}
$$

Please note that in the above derivation process, it is impossible to obtain the best weights $p_{i}$ by making $\left.\frac{\partial\left(\sigma^{2}(V)\right)}{\partial P_{i}}\right|_{P_{i}=p_{i}}=0$, because the best weight $p_{i}$ actually depends on the evaluation $\sigma^{2}\left(\Delta x_{i}\right)$ of error $\Delta x_{i}=x_{i}-X_{T}$ rather than the evaluation $\sigma^{2}(V)$ of error $v_{i}=x_{i}-y$.

For the convenience of calculation, making $\sum_{i=1}^{n} P_{i}=1$, the formula (3-5) becomes:

$$
y=P_{1} x_{1}+P_{2} x_{2}+\cdots+P_{n} x_{n}
$$

Now, using the quality of each $x_{i}$, we begin to solve the general calculation method of each weight $p_{i}$.

The error propagation equation is obtained by taking the total differential of equation (3-6), that is:

$$
\Delta y=P_{1} \Delta x_{1}+P_{2} \Delta x_{2}+\cdots+P_{n} \Delta x_{n}
$$

Applying the formula (2-17) to equation (3-7), there is: 


$$
\begin{aligned}
& \sigma^{2}(\Delta y)=\left[\begin{array}{llll}
P_{1} & P_{2} & \cdots & P_{n}
\end{array}\right] \mathbf{D}(\Delta \mathbf{X})\left[\begin{array}{c}
P_{1} \\
P_{2} \\
\vdots \\
P_{n}
\end{array}\right] \\
& =P_{1}^{2} \sigma_{1}^{2}+P_{2}^{2} \sigma_{2}^{2}+\cdots+P_{n}^{2} \sigma_{n}^{2}+2 P_{1} P_{2} \sigma_{12}+2 P_{1} P_{3} \sigma_{13}+\cdots
\end{aligned}
$$

Please note that the variance $\sigma_{i}^{2}$ is the dispersion $\sigma^{2}\left(\Delta x_{i}\right)$ of all possible values of the error $\Delta x_{i}=x_{i}-X_{T}$, which expresses the evaluation of the probability interval of the error $\Delta x_{i}$. This concept of variance is consistent with probability theory, but it is different from the concept of dispersion $\sigma^{2}\left(x_{i}\right)$ of observation $x_{i}$ in traditional measurement theory.

When the value of the function $\sigma^{2}(\Delta y)$ is the minimum, the quality of the measured value $y$ is the best, which is a problem of finding the minimum value of the function $\sigma^{2}(\Delta y)$ under the restriction condition $\sum_{i=1}^{n} P_{i}=1$. Therefore, according to the Lagrange multiplier method, the following functions are formed:

$$
\begin{aligned}
L a & =\sigma^{2}(\Delta y)+2 \lambda\left(P_{1}+P_{2}+\cdots+P_{n}-1\right) \\
& =P_{1}^{2} \sigma_{1}^{2}+P_{2}^{2} \sigma_{2}^{2}+\cdots+P_{n}^{2} \sigma_{n}^{2}+2 P_{1} P_{2} \sigma_{12}+2 P_{1} P_{3} \sigma_{13}+\cdots \\
& +2 \lambda\left(P_{1}+P_{2}+\cdots+P_{n}-1\right)
\end{aligned}
$$

Taking the partial derivative of each $P_{i}$ and making it equal to 0 , the Lagrange equation is obtained as follows:

$$
\left.\begin{array}{rc}
\left.\left(P_{1} \sigma_{1}^{2}+P_{2} \sigma_{12}+P_{3} \sigma_{13}+\cdots+P_{n} \sigma_{1 n}+\lambda\right)\right|_{P_{i}=p_{i}, \lambda=\lambda_{0}}=0 \\
\left.\left(P_{1} \sigma_{21}+P_{2} \sigma_{2}^{2}+P_{3} \sigma_{23}+\cdots+P_{n} \sigma_{2 n}+\lambda\right)\right|_{P_{i}=p_{i}, \lambda=\lambda_{0}}=0 \\
\vdots \\
\left.\left(P_{1} \sigma_{n 1}+P_{2} \sigma_{n 2}+P_{3} \sigma_{n 3}+\cdots+P_{n} \sigma_{n}^{2}+\lambda\right)\right|_{P_{i}=p_{i}, \lambda=\lambda_{0}}=0 \\
\left.\left(P_{1}+P_{2}+\cdots+P_{n}-1\right)\right|_{P_{i}=p_{i}, \lambda=\lambda_{0}}=0
\end{array}\right\}
$$

That is:

$$
\left[\begin{array}{ccccc}
\sigma_{1}^{2} & \sigma_{12} & \cdots & \sigma_{1 n} & 1 \\
\sigma_{21} & \sigma_{2}^{2} & \cdots & \sigma_{2 n} & 1 \\
\vdots & \vdots & \ddots & \vdots & \vdots \\
\sigma_{n 1} & \sigma_{n 2} & \cdots & \sigma_{n}^{2} & 1 \\
1 & 1 & \cdots & 1 & 0
\end{array}\right]\left[\begin{array}{c}
p_{1} \\
p_{2} \\
\vdots \\
p_{n} \\
\lambda_{0}
\end{array}\right]=\left[\begin{array}{c}
0 \\
0 \\
\vdots \\
0 \\
1
\end{array}\right]
$$

Because the covariance array of error array $\Delta \mathbf{X}=\left[\Delta x_{1}, \Delta x_{2}, \cdots, \Delta x_{n}\right]^{\mathrm{T}}$ is: 


$$
\mathbf{D}(\boldsymbol{\Delta} \mathbf{X})=E\left[\begin{array}{c}
\Delta x_{1} \\
\Delta x_{2} \\
\vdots \\
\Delta x_{n}
\end{array}\right]\left[\begin{array}{llll}
\Delta x_{1} & \Delta x_{2} & \cdots & \Delta x_{n}
\end{array}\right]=\left[\begin{array}{cccc}
\sigma_{1}^{2} & \sigma_{12} & \cdots & \sigma_{1 n} \\
\sigma_{21} & \sigma_{2}^{2} & \cdots & \sigma_{2 n} \\
\vdots & \vdots & \vdots & \vdots \\
\sigma_{n 1} & \sigma_{n 2} & \cdots & \sigma_{n}^{2}
\end{array}\right]
$$

So the equation (3-11) can be written as follows:

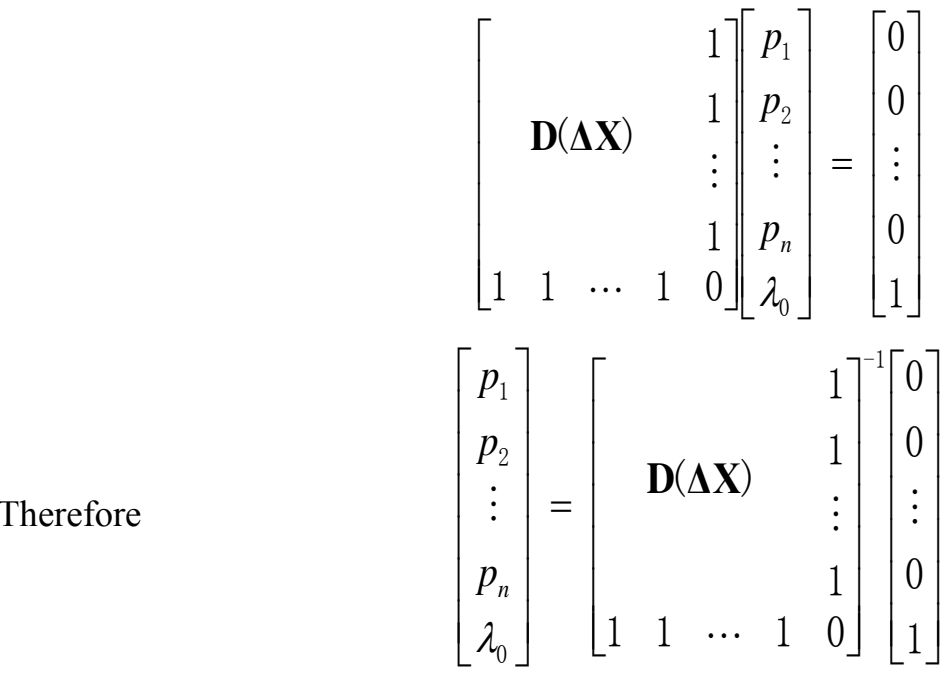

In this way, according to formula (3-6), the optimal measured value is

$$
\begin{aligned}
y_{0} & =\left.y\right|_{P_{i}=p_{i}} \\
& =p_{1} x_{1}+p_{2} x_{2}+\cdots+p_{n} x_{n}
\end{aligned}
$$

And according to formula (3-13), there is

$$
\mathbf{D}(\Delta \mathbf{X})\left[\begin{array}{c}
p_{1} \\
p_{2} \\
\vdots \\
p_{n}
\end{array}\right]+\left[\begin{array}{c}
1 \\
1 \\
\vdots \\
1
\end{array}\right]\left[\lambda_{0}\right]=\left[\begin{array}{c}
0 \\
0 \\
\vdots \\
0
\end{array}\right]
$$

Therefore

$$
\left[\begin{array}{llll}
p_{1} & p_{2} & \cdots & p_{n}
\end{array}\right] \mathbf{D}(\boldsymbol{\Delta} \mathbf{X})\left[\begin{array}{c}
p_{1} \\
p_{2} \\
\vdots \\
p_{n}
\end{array}\right]=-\lambda_{0}
$$

According to equation (3-8), we can get:

$$
\begin{aligned}
\sigma^{2}\left(\Delta y_{0}\right) & =\left.\sigma^{2}(\Delta y)\right|_{P_{i}=p_{i}} \\
& =\left[\begin{array}{llll}
p_{1} & p_{2} & \cdots & p_{n}
\end{array}\right] \mathbf{D}(\Delta \mathbf{X})\left[\begin{array}{c}
p_{1} \\
p_{2} \\
\vdots \\
p_{n}
\end{array}\right] \\
& =-\lambda_{0}
\end{aligned}
$$


In formula (3-11), if each $\sigma_{i j}=0$, we can get the following results:

$$
p_{1}: p_{2}: \cdots: p_{n}=\frac{1}{\sigma_{1}^{2}}: \frac{1}{\sigma_{2}^{2}}: \cdots: \frac{1}{\sigma_{n}^{2}}
$$

This is consistent with the fact that we used to use a single $\frac{1}{\sigma_{i}^{2}}$ to determine the weight ratio. Obviously, the formula (3-19) does not hold when the hypothesis $\sigma_{i j}=0$ does not exist.

Next, let's see the actual cases.

Example 1, as shown in Fig 2, when $n$ observations $x_{i}$ are obtained by using the same sensor to directly repeat the measurement, please calculate the final measured value according to formula (3-6).

Because $\Delta x_{i}=\Delta_{B}+\Delta_{A i}$, so according to the formula (2-11), there is:

$$
\sigma_{i}^{2}=\sigma_{\Delta_{B}}^{2}+\sigma_{\Delta_{A}}^{2}
$$

For any two observation errors $\Delta x_{j}$ and $\Delta x_{k}$, according to the formula (2-15), there is:

$$
\begin{aligned}
\sigma_{j k} & =E\left(\Delta x_{j} \Delta x_{k}\right) \\
& =E\left[\left(\Delta_{B}+\Delta_{A j}\right)\left(\Delta_{B}+\Delta_{A k}\right)\right] \\
& =E\left(\Delta_{B}^{2}\right)=\sigma_{\Delta_{B}}^{2}
\end{aligned}
$$

Therefore, according to the formula (3-11), there is:

$$
\left[\begin{array}{ccccc}
\sigma_{\Delta_{B}}^{2}+\sigma_{\Delta_{A}}^{2} & \sigma_{\Delta_{B}}^{2} & \cdots & \sigma_{\Delta_{B}}^{2} & 1 \\
\sigma_{\Delta_{B}}^{2} & \sigma_{\Delta_{B}}^{2}+\sigma_{\Delta_{A}}^{2} & \cdots & \sigma_{\Delta_{B}}^{2} & 1 \\
\vdots & \vdots & \ddots & \vdots & \vdots \\
\sigma_{\Delta_{B}}^{2} & \sigma_{\Delta_{B}}^{2} & \cdots & \sigma_{\Delta_{B}}^{2}+\sigma_{\Delta_{A}}^{2} & 1 \\
1 & 1 & \cdots & 1 & 0
\end{array}\right]\left[\begin{array}{c}
p_{1} \\
p_{2} \\
\vdots \\
p_{n} \\
\lambda_{0}
\end{array}\right]=\left[\begin{array}{c}
0 \\
0 \\
\vdots \\
0 \\
1
\end{array}\right]
$$

By solving the equation, we get the following results:

$$
p_{1}=p_{2}=\cdots=p_{n}=\frac{1}{n} \quad \lambda_{0}=-\sigma_{\Delta_{B}}^{2}-\frac{\sigma_{\Delta_{A}}^{2}}{n}
$$

Finally, according to (3-15) and (3-18), there are:

$$
y_{0}=\frac{x_{1}+x_{2}+\cdots+x_{n}}{n}, \quad \sigma^{2}\left(\Delta y_{0}\right)=\sigma_{\Delta_{B}}^{2}+\frac{\sigma_{\Delta_{A}}^{2}}{n} .
$$

It can be seen that in this case, the root cause of $p_{1}=p_{2}=\cdots=p_{n}=\frac{1}{n}$ is that each error $\Delta x_{i}$ has the same variance $\sigma_{i}^{2}=\sigma_{\Delta_{B}}^{2}+\sigma_{\Delta_{A}}^{2}$ and any two errors $\Delta x_{i}$ and $\Delta x_{j}$ have the same covariance $\sigma_{j k}=\sigma_{\Delta_{B}}^{2}$. Moreover, it can be seen that the traditional measurement theory classifies $\Delta_{B}$ as systematic error, which is actually to negate the correlation between $\Delta x_{i}$ and $\Delta x_{j}$.

Table 3. Observed values 


\begin{tabular}{|c|c|c|c|c|}
\hline$i$ & 1 & 2 & 3 & 4 \\
\hline$a_{i}$ & 5 & 10 & 15 & 20 \\
\hline$l_{i}(\mathrm{~mm})$ & 2.87 & 5.72 & 8.60 & 11.37 \\
\hline
\end{tabular}

Example 2, as shown in Fig 4, in order to obtain the accurate diameter value of the copper wire, the observed values of 5 times, 10 times, 15 times and 20 times of the diameter are measured with a caliper, and 4 observed values are shown in Table 3. Please give the best measured value of diameter and the evaluation of uncertainty.

The four measured values are as follows:

$$
\begin{aligned}
& x_{1}=\frac{l_{1}}{a_{1}}=0.574 \\
& x_{2}=\frac{l_{2}}{a_{2}}=0.572 \\
& x_{3}=\frac{l_{3}}{a_{3}}=0.5733 \\
& x_{4}=\frac{l_{4}}{a_{4}}=0.5685
\end{aligned}
$$

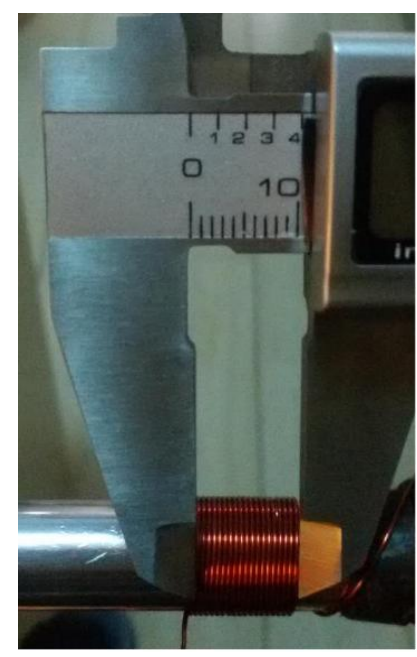

Fig 4. Measuring the diameter of copper wire

The error $\Delta l_{i}$ of the observed value $l_{i}$ consists of three components: fixed error $K$, proportional error $R$ and non-uniform dividing error $c_{i}$. That is:

$$
\Delta l_{i}=K+l_{i} R+c_{i}
$$

According to the definition (2-16) of variance, there is

$$
\begin{aligned}
\mathbf{D}(\Delta \mathbf{L}) & =E\left[\begin{array}{llll}
\Delta l_{1} & \Delta l_{2} & \Delta l_{3} & \Delta l_{4}
\end{array}\right]\left[\begin{array}{c}
\Delta l_{1} \\
\Delta l_{2} \\
\Delta l_{3} \\
\Delta l_{4}
\end{array}\right] \\
& =\left[\begin{array}{llll}
\sigma_{K}^{2}+l_{1}^{2} \sigma_{R}^{2}+\sigma_{c}^{2} & \sigma_{K}^{2}+l_{2} l_{1} \sigma_{R}^{2} & \sigma_{K}^{2}+l_{3} l_{1} \sigma_{R}^{2} & \sigma_{K}^{2}+l_{4} l_{1} \sigma_{R}^{2} \\
\sigma_{K}^{2}+l_{1} l_{2} \sigma_{R}^{2} & \sigma_{K}^{2}+l_{2}^{2} \sigma_{R}^{2}+\sigma_{c}^{2} & \sigma_{K}^{2}+l_{3} l_{2} \sigma_{R}^{2} & \sigma_{K}^{2}+l_{4} l_{2} \sigma_{R}^{2} \\
\sigma_{K}^{2}+l_{1} l_{3} \sigma_{R}^{2} & \sigma_{K}^{2}+l_{2} l_{3} \sigma_{R}^{2} & \sigma_{K}^{2}+l_{3}^{2} \sigma_{R}^{2}+\sigma_{c}^{2} & \sigma_{K}^{2}+l_{4} l_{3} \sigma_{R}^{2} \\
\sigma_{K}^{2}+l_{1} l_{4} \sigma_{R}^{2} & \sigma_{K}^{2}+l_{2} l_{4} \sigma_{R}^{2} & \sigma_{K}^{2}+l_{3} l_{4} \sigma_{R}^{2} & \sigma_{K}^{2}+l_{4}^{2} \sigma_{R}^{2}+\sigma_{c}^{2}
\end{array}\right]
\end{aligned}
$$

Because $x_{i}=l_{i} / a_{i}$ and $\Delta x_{i}=\Delta l_{i} / a_{i}$, there is

$$
\left[\begin{array}{l}
\Delta x_{1} \\
\Delta x_{2} \\
\Delta x_{3} \\
\Delta x_{4}
\end{array}\right]=\left[\begin{array}{cccc}
a_{1}^{-1} & 0 & 0 & 0 \\
0 & a_{2}^{-1} & 0 & 0 \\
0 & 0 & a_{3}^{-1} & 0 \\
0 & 0 & 0 & a_{4}^{-1}
\end{array}\right]\left[\begin{array}{c}
\Delta l_{1} \\
\Delta l_{2} \\
\Delta l_{3} \\
\Delta l_{4}
\end{array}\right]
$$

That is

$$
\Delta \mathbf{X}=\mathbf{A} \Delta \mathbf{L}
$$

According to the formula (2-17), there is:

$\mathbf{D}(\Delta \mathbf{X})=\mathbf{A D}(\Delta \mathbf{L}) \mathbf{A}^{\mathrm{T}}$

$$
=\left[\begin{array}{llll}
a_{1}^{-2}\left(\sigma_{K}^{2}+l_{1}^{2} \sigma_{R}^{2}+\sigma_{c}^{2}\right) & a_{1}^{-1} a_{2}^{-1}\left(\sigma_{K}^{2}+l_{2} l_{1} \sigma_{R}^{2}\right) & a_{1}^{-1} a_{3}^{-1}\left(\sigma_{K}^{2}+l_{3} l_{1} \sigma_{R}^{2}\right) & a_{1}^{-1} a_{4}^{-1}\left(\sigma_{K}^{2}+l_{4} l_{1} \sigma_{R}^{2}\right) \\
a_{2}^{-1} a_{1}^{-1}\left(\sigma_{K}^{2}+l_{1} l_{2} \sigma_{R}^{2}\right) & a_{2}^{-2}\left(\sigma_{K}^{2}+l_{2}^{2} \sigma_{R}^{2}+\sigma_{c}^{2}\right) & a_{2}^{-1} a_{3}^{-1}\left(\sigma_{K}^{2}+l_{3} l_{2} \sigma_{R}^{2}\right) & a_{2}^{-1} a_{4}^{-1}\left(\sigma_{K}^{2}+l_{4} l_{2} \sigma_{R}^{2}\right) \\
a_{3}^{-1} a_{1}^{-1}\left(\sigma_{K}^{2}+l_{1} l_{3} \sigma_{R}^{2}\right) & a_{3}^{-1} a_{2}^{-1}\left(\sigma_{K}^{2}+l_{2} l_{3} \sigma_{R}^{2}\right) & a_{3}^{-2}\left(\sigma_{K}^{2}+l_{3}^{2} \sigma_{R}^{2}+\sigma_{c}^{2}\right) & a_{3}^{-1} a_{4}^{-1}\left(\sigma_{K}^{2}+l_{4} l_{3} \sigma_{R}^{2}\right) \\
a_{4}^{-1} a_{1}^{-1}\left(\sigma_{K}^{2}+l_{1} l_{4} \sigma_{R}^{2}\right) & a_{4}^{-1} a_{2}^{-1}\left(\sigma_{K}^{2}+l_{2} l_{4} \sigma_{R}^{2}\right) & a_{4}^{-1} a_{3}^{-1}\left(\sigma_{K}^{2}+l_{3} l_{4} \sigma_{R}^{2}\right) & a_{4}^{-2}\left(\sigma_{K}^{2}+l_{4}^{2} \sigma_{R}^{2}+\sigma_{c}^{2}\right)
\end{array}\right]
$$


Assuming $\sigma_{K}= \pm 0.01 \mathrm{~mm}, \sigma_{R}= \pm 1 \times 10^{-5}$ and $\sigma_{c}= \pm 0.02 \mathrm{~mm}$, there is:

$$
\mathbf{D}(\boldsymbol{\Delta} \mathbf{X})=\left[\begin{array}{cccc}
2 \times 10^{-5} & 2 \times 10^{-6} & 1.33333 \times 10^{-6} & 1 \times 10^{-6} \\
2 \times 10^{-6} & 5 \times 10^{-6} & 6.66667 \times 10^{-7} & 5 \times 10^{-7} \\
1.33333 \times 10^{-6} & 6.66667 \times 10^{-7} & 2.22222 \times 10^{-6} & 3.33334 \times 10^{-7} \\
1 \times 10^{-6} & 5 \times 10^{-7} & 3.33334 \times 10^{-7} & 1.25 \times 10^{-6}
\end{array}\right]
$$

According to the formula (3-14), there is:

$$
\left[\begin{array}{l}
p_{1} \\
p_{2} \\
p_{3} \\
p_{4} \\
\lambda_{0}
\end{array}\right]=\left[\begin{array}{c}
-0.014285714 \\
0.085714286 \\
0.299999996 \\
0.628571432 \\
-9.14286 \times 10^{-7}
\end{array}\right]
$$

According to the formula (3-15), there is:

$$
y_{0}=\left[\begin{array}{llll}
p_{1} & p_{2} & p_{3} & p_{4}
\end{array}\left[\begin{array}{l}
x_{1} \\
x_{2} \\
x_{3} \\
x_{4}
\end{array}\right]=0.5702(\mathrm{~mm})\right.
$$

According to the formula (3-18), there is:

$$
\begin{aligned}
\sigma^{2}\left(\Delta y_{0}\right) & =-\lambda_{0} \\
& =9.14286 \times 10^{-7}\left(\mathrm{~mm}^{2}\right)
\end{aligned}
$$

Therefore, the uncertainty is:

$$
\sigma\left(\Delta y_{0}\right) \approx \pm 0.00096(\mathrm{~mm})
$$

As you can see, $p_{1}$ is a negative value, which is beyond our inherent concept. In the past, we used to follow the conceptual logic of traditional theory to determine weights according to precision, ignoring the correlation between errors, so that $\mathbf{D}(\Delta \mathbf{X})$ is a diagonal matrix, and $p_{i}$ is always positive. Now, variance is the dispersion of all possible values of error, and any error has variance and covariance, so $\mathbf{D}(\Delta \mathbf{X})$ is no longer a diagonal matrix, and $p_{i}$ can be negative.

However, this negative weight is also a positive contribution to the final measured value. In this case, $a_{1}$ is the smallest, and the fixed error $K$ is too harmful to the observed value $x_{1}$. Therefore, Lagrange algorithm can only choose negative weights to correct this harm, which is exactly the scientific point of this algorithm.

\subsection{Indirect measurement model for single measurand}

The observation error equation is as follows.

$$
\left[\begin{array}{c}
P_{1}^{1 / 2} V_{1} \\
P_{2}^{1 / 2} V_{2} \\
\vdots \\
P_{n}^{1 / 2} V_{n}
\end{array}\right]=\left[\begin{array}{c}
P_{1}^{1 / 2} x_{1} \\
P_{2}^{1 / 2} x_{2} \\
\vdots \\
P_{n}^{1 / 2} x_{n}
\end{array}\right]-\left[\begin{array}{cccc}
P_{1}^{1 / 2} & 0 & \cdots & 0 \\
0 & P_{2}^{1 / 2} & \cdots & 0 \\
\vdots & \vdots & \vdots & \vdots \\
0 & 0 & \cdots & P_{n}^{1 / 2}
\end{array}\right]\left[\begin{array}{c}
a_{1} \\
a_{2} \\
\vdots \\
a_{n}
\end{array}\right][Y]
$$

According to the least square method, there is:

$$
y=\frac{\sum P_{i} a_{i} x_{i}}{\sum P_{i} a_{i}^{2}}
$$

Similar to the above direct measurement model, in order to realize the minimum value of 
$\sigma^{2}(\Delta y)$, it is assumed that $\sum P_{i} a_{i}^{2}=1$, and the formula (3-21) becomes:

$$
\begin{aligned}
y & =P_{1} a_{1} x_{1}+P_{2} a_{2} x_{2}+\cdots+P_{n} a_{n} x_{n} \\
& =\left[\begin{array}{llll}
a_{1} & a_{2} & \cdots & a_{n}
\end{array}\left[\begin{array}{cccc}
P_{1} & 0 & \cdots & 0 \\
0 & P_{2} & \cdots & 0 \\
\vdots & \vdots & & \vdots \\
0 & 0 & \cdots & P_{n}
\end{array}\right]\left[\begin{array}{c}
x_{1} \\
x_{2} \\
\vdots \\
x_{n}
\end{array}\right]\right.
\end{aligned}
$$

That is

$$
y=\mathbf{A}^{\mathrm{T}} \mathbf{P X}
$$

$$
\text { Among them, } \quad \mathbf{A}=\left[\begin{array}{c}
a_{1} \\
a_{2} \\
\vdots \\
a_{n}
\end{array}\right], \mathbf{P}=\left[\begin{array}{cccc}
P_{1} & 0 & \cdots & 0 \\
0 & P_{2} & \cdots & 0 \\
\vdots & \vdots & & \vdots \\
0 & 0 & \cdots & P_{n}
\end{array}\right], \mathbf{X}=\left[\begin{array}{c}
x_{1} \\
x_{2} \\
\vdots \\
x_{n}
\end{array}\right] \text {. }
$$

The error propagation equation is obtained by taking the total differential of formula (3-23), that is:

$$
\Delta y=\mathbf{A}^{\mathrm{T}} \mathbf{P} \Delta \mathbf{X}
$$

Applying to the formula (2-17) to equation (3-24), there is:

$$
\begin{aligned}
\sigma^{2}(\Delta y) & =\mathbf{A}^{\mathrm{T}} \mathbf{P D}(\boldsymbol{\Delta} \mathbf{X}) \mathbf{P A} \\
& =P_{1}^{2} a_{1}^{2} \sigma_{1}^{2}+P_{2}^{2} a_{2}^{2} \sigma_{2}^{2}+\cdots+P_{n}^{2} a_{n}^{2} \sigma_{n}^{2} \\
& +2 P_{1} P_{2} a_{1} a_{2} \sigma_{12}+2 P_{1} P_{3} a_{1} a_{3} \sigma_{13}+\cdots
\end{aligned}
$$

Similarly, according to Lagrange multiplier method and omitting the derivation process, it is concluded that:

$$
\begin{aligned}
& {\left[\begin{array}{ccccc}
a_{1}^{2} \sigma_{1}^{2} & a_{1} a_{2} \sigma_{12} & \cdots & a_{1} a_{n} \sigma_{1 n} & a_{1}^{2} \\
a_{2} a_{1} \sigma_{21} & a_{2}^{2} \sigma_{2}^{2} & \cdots & a_{2} a_{n} \sigma_{2 n} & a_{2}^{2} \\
\vdots & \vdots & \ddots & \vdots & \vdots \\
a_{n} a_{1} \sigma_{n 1} & a_{n} a_{2} \sigma_{n 2} & \cdots & a_{n}^{2} \sigma_{n}^{2} & a_{n}^{2} \\
a_{1}^{2} & a_{2}^{2} & \cdots & a_{n}^{2} & 0
\end{array}\right]\left[\begin{array}{c}
p_{1} \\
p_{2} \\
\vdots \\
p_{n} \\
\lambda_{0}
\end{array}\right]=\left[\begin{array}{c}
0 \\
0 \\
\vdots \\
0 \\
1
\end{array}\right]} \\
& \text { Making } \mathbf{A}^{\prime}=\left[\begin{array}{cccc}
a_{1} & 0 & \cdots & 0 \\
0 & a_{2} & \cdots & 0 \\
\vdots & \vdots & & \vdots \\
0 & 0 & \cdots & a_{n}
\end{array}\right] \text {, the formula (3-26) becomes: } \\
& {\left[\begin{array}{c}
p_{1} \\
p_{2} \\
\vdots \\
p_{n} \\
\lambda_{0}
\end{array}\right]=\left[\begin{array}{ccccc} 
& & & a_{1}^{2} \\
& & & a_{2}^{2} \\
\mathbf{A}^{\prime} \mathbf{D}(\Delta \mathbf{X}) \mathbf{A}^{\prime} & & \vdots \\
& & & a_{n}^{2} \\
a_{1}^{2} & a_{2}^{2} & \cdots & a_{n}^{2} & 0
\end{array}\right]^{-1}\left[\begin{array}{c}
0 \\
0 \\
\vdots \\
0 \\
1
\end{array}\right]}
\end{aligned}
$$

It can be seen that the best weights are actually determined by $\mathbf{D}(\Delta \mathbf{X})$ and $\mathbf{A}^{\prime}$ together. In this way, according to formula (3-23), the optimal measured value is 


$$
\begin{aligned}
y_{0} & =\left.y\right|_{P_{i}=p_{i}} \\
& =\left.\mathbf{A}^{\mathrm{T}} \mathbf{P X}\right|_{P_{i}=p_{i}}
\end{aligned}
$$

And according to (3-27), there is:

$$
\mathbf{A}^{\prime} \mathbf{D}(\Delta \mathbf{X}) \mathbf{A}^{\prime}\left[\begin{array}{c}
p_{1} \\
p_{2} \\
\vdots \\
p_{n}
\end{array}\right]=\left[\begin{array}{c}
a_{1}^{2} \\
a_{2}^{2} \\
\vdots \\
a_{n}^{2}
\end{array}\right]\left[-\lambda_{0}\right]
$$

So there is

$$
\begin{aligned}
{\left[\begin{array}{llll}
p_{1} & p_{2} & \cdots & p_{n}
\end{array}\right] \mathbf{A}^{\prime} \mathbf{D}(\Delta \mathbf{X}) \mathbf{A}^{\prime}\left[\begin{array}{c}
p_{1} \\
p_{2} \\
\vdots \\
p_{n}
\end{array}\right] } & =\left[\begin{array}{llll}
p_{1} & p_{2} & \cdots & p_{n}
\end{array}\right]\left[\begin{array}{c}
a_{1}^{2} \\
a_{2}^{2} \\
\vdots \\
a_{n}^{2}
\end{array}\right]\left[\begin{array}{l}
-\lambda_{0}
\end{array}\right] \\
& =-\lambda_{0}
\end{aligned}
$$

And because there is:

$$
\begin{aligned}
\left.\mathbf{A}^{\mathrm{T}} \mathbf{P D}(\Delta \mathbf{X}) \mathbf{P A}\right|_{P_{i}=p_{i}} & =\left[\begin{array}{llll}
p_{1} & p_{2} & \cdots & p_{n}
\end{array}\right] \mathbf{A}^{\prime} \mathbf{D}(\Delta \mathbf{X}) \mathbf{A}^{\prime}\left[\begin{array}{c}
p_{1} \\
p_{2} \\
\vdots \\
p_{n}
\end{array}\right] \\
& =-\lambda_{0}
\end{aligned}
$$

So according to formula (3-25), there is:

$$
\begin{aligned}
\sigma^{2}\left(\Delta y_{0}\right) & =\left.\sigma^{2}(\Delta y)\right|_{P_{i}=p_{i}} \\
& =\left.\mathbf{A}^{\mathrm{T}} \mathbf{P D}(\Delta \mathbf{X}) \mathbf{P A}\right|_{P_{i}=p_{i}} \\
& =-\lambda_{0}
\end{aligned}
$$

Example 3, the data of example 2 is still used, and the solution is carried out according to the (3-19) model.

The observation error equation is as follows.

$$
\begin{aligned}
& {\left[\begin{array}{l}
P_{1}^{1 / 2} V_{1} \\
P_{2}^{1 / 2} V_{2} \\
P_{3}^{1 / 2} V_{3} \\
P_{4}^{1 / 2} V_{4}
\end{array}\right]=\left[\begin{array}{l}
P_{1}^{1 / 2} l_{1} \\
P_{2}^{1 / 2} l_{2} \\
P_{3}^{1 / 2} l_{3} \\
P_{4}^{1 / 2} l_{4}
\end{array}\right]-\left[\begin{array}{cccc}
P_{1}^{1 / 2} & 0 & 0 & 0 \\
0 & P_{2}^{1 / 2} & 0 & 0 \\
0 & 0 & P_{3}^{1 / 2} & 0 \\
0 & 0 & 0 & P_{4}^{1 / 2}
\end{array}\right]\left[\begin{array}{l}
a_{1} \\
a_{2} \\
a_{3} \\
a_{4}
\end{array}\right][Y] } \\
& \text { There are } \mathbf{L}=\left[\begin{array}{l}
l_{1} \\
l_{2} \\
l_{3} \\
l_{4}
\end{array}\right]=\left[\begin{array}{c}
2.87 \\
5.72 \\
8.6 \\
11.37
\end{array}\right], \mathbf{A}=\left[\begin{array}{l}
a_{1} \\
a_{2} \\
a_{3} \\
a_{4}
\end{array}\right]=\left[\begin{array}{c}
5 \\
10 \\
15 \\
20
\end{array}\right] \text { and } \mathbf{A}^{\prime}=\left[\begin{array}{cccc}
5 & 0 & 0 & 0 \\
0 & 10 & 0 & 0 \\
0 & 0 & 15 & 0 \\
0 & 0 & 0 & 20
\end{array}\right] .
\end{aligned}
$$


Because

$$
\Delta l_{i}=K+l_{i} R+c_{i}
$$

According to the definition (2-16) of variance, there is

$$
\begin{gathered}
\mathbf{D}(\boldsymbol{\Delta} \mathbf{L})=E\left[\begin{array}{llll}
\Delta l_{1} & \Delta l_{2} & \Delta l_{3} & \Delta l_{4}
\end{array}\right]\left[\begin{array}{c}
\Delta l_{1} \\
\Delta l_{2} \\
\Delta l_{3} \\
\Delta l_{4}
\end{array}\right] \\
=\left[\begin{array}{llll}
\sigma_{K}^{2}+l_{1}^{2} \sigma_{R}^{2}+\sigma_{c}^{2} & \sigma_{K}^{2}+l_{2} l_{1} \sigma_{R}^{2} & \sigma_{K}^{2}+l_{3} l_{1} \sigma_{R}^{2} & \sigma_{K}^{2}+l_{4} l_{1} \sigma_{R}^{2} \\
\sigma_{K}^{2}+l_{1} l_{2} \sigma_{R}^{2} & \sigma_{K}^{2}+l_{2}^{2} \sigma_{R}^{2}+\sigma_{c}^{2} & \sigma_{K}^{2}+l_{3} l_{2} \sigma_{R}^{2} & \sigma_{K}^{2}+l_{4} l_{2} \sigma_{R}^{2} \\
\sigma_{K}^{2}+l_{1} l_{3} \sigma_{R}^{2} & \sigma_{K}^{2}+l_{2} l_{3} \sigma_{R}^{2} & \sigma_{K}^{2}+l_{3}^{2} \sigma_{R}^{2}+\sigma_{c}^{2} & \sigma_{K}^{2}+l_{4} l_{3} \sigma_{R}^{2} \\
\sigma_{K}^{2}+l_{1} l_{4} \sigma_{R}^{2} & \sigma_{K}^{2}+l_{2} l_{4} \sigma_{R}^{2} & \sigma_{K}^{2}+l_{3} l_{4} \sigma_{R}^{2} & \sigma_{K}^{2}+l_{4}^{2} \sigma_{R}^{2}+\sigma_{c}^{2}
\end{array}\right]
\end{gathered}
$$

Substituting $\sigma_{K}= \pm 0.01 \mathrm{~mm}, \sigma_{R}= \pm 1 \times 10^{-5}$ and $\sigma_{c}= \pm 0.02 \mathrm{~mm}$, and according to the formula (3-27), there is:

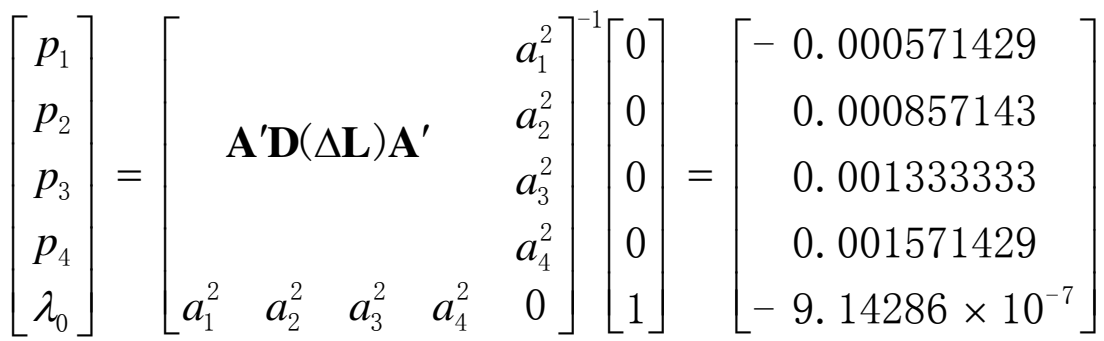

According to formula (3-28), the best measured value is as follows:

$$
y_{0}=\left.\mathbf{A}^{\mathrm{T}} \mathbf{P X}\right|_{P_{i}=p_{i}}=0.5702(\mathrm{~mm})
$$

According to the formula (3-32), there is:

$$
\begin{aligned}
\sigma^{2}\left(\Delta y_{0}\right) & =-\lambda_{0} \\
& =9.14286 \times 10^{-7}\left(\mathrm{~mm}^{2}\right)
\end{aligned}
$$

Therefore, the uncertainty is $\sigma\left(\Delta y_{0}\right)= \pm 0.00096(\mathrm{~mm})$

It can be seen that the final results of example 3 and example 2 are the same.

\subsection{Indirect measurement model for multiple measurands}

The observation error equation is as follows.

$$
\left[\begin{array}{c}
P_{1}^{1 / 2} V_{1} \\
P_{2}^{1 / 2} V_{2} \\
\vdots \\
P_{n}^{1 / 2} V_{n}
\end{array}\right]=\left[\begin{array}{c}
P_{1}^{1 / 2} x_{1} \\
P_{2}^{1 / 2} x_{2} \\
\vdots \\
P_{n}^{1 / 2} x_{n}
\end{array}\right]-\left[\begin{array}{cccc}
P_{1}^{1 / 2} & 0 & \cdots & 0 \\
0 & P_{2}^{1 / 2} & \cdots & 0 \\
\vdots & \vdots & \vdots & \vdots \\
0 & 0 & \cdots & P_{n}^{1 / 2}
\end{array}\right]\left[\begin{array}{cccc}
a_{11} & a_{12} & \cdots & a_{1 t} \\
a_{21} & a_{22} & \cdots & a_{2 t} \\
\vdots & \vdots & \vdots & \vdots \\
a_{n 1} & a_{n 2} & \cdots & a_{n t}
\end{array}\right]\left[\begin{array}{c}
Y_{1} \\
Y_{2} \\
\vdots \\
Y_{t}
\end{array}\right]
$$

According to the least square method, there is:

$$
\begin{gathered}
\mathbf{Y}=\left[\mathbf{A}^{\mathbf{T}} \mathbf{P A}\right]^{-1} \mathbf{A}^{\mathbf{T}} \mathbf{P X} \\
\mathbf{P}=\left[\begin{array}{cccc}
P_{1} & 0 & \cdots & 0 \\
0 & P_{2} & \cdots & 0 \\
\vdots & \vdots & \vdots & \vdots \\
0 & 0 & \cdots & P_{n}
\end{array}\right], \mathbf{A}=\left[\begin{array}{cccc}
a_{11} & a_{12} & \cdots & a_{1 t} \\
a_{21} & a_{22} & \cdots & a_{2 t} \\
\vdots & \vdots & \vdots & \vdots \\
a_{n 1} & a_{n 2} & \cdots & a_{n t}
\end{array}\right], \mathbf{Y}=\left[\begin{array}{c}
y_{1} \\
y_{2} \\
\vdots \\
y_{t}
\end{array}\right], \mathbf{X}=\left[\begin{array}{c}
x_{1} \\
x_{2} \\
\vdots \\
x_{n}
\end{array}\right]
\end{gathered}
$$

The error propagation equation is obtained by taking the total differential of formula (3-34), that is: 


$$
\Delta \mathbf{Y}=\left[\mathbf{A}^{\mathrm{T}} \mathbf{P A}\right]^{-1} \mathbf{A}^{\mathrm{T}} \mathbf{P} \Delta \mathbf{X}
$$

Applying the law of covariance propagation to formula (3-35), we get:

$$
\mathbf{D}(\Delta \mathbf{Y})=\left[\mathbf{A}^{\mathrm{T}} \mathbf{P A}\right]^{-1} \mathbf{A}^{\mathrm{T}} \mathbf{P D}(\Delta \mathbf{X}) \mathbf{P A}\left[\mathbf{A}^{\mathrm{T}} \mathbf{P A}\right]^{-1}
$$

However, in the error propagation equation (3-35), the propagation law of each error $\Delta y_{j}$ is different from each other, so it is difficult to ensure that the variance of all errors can be minimized at the same time. This is also a topic worthy of further study by readers, and the authors consider this topic as follows.

There are $t$ output errors $\Delta y_{j}$ in the formula (3-35), and we hope their weighted average error $\Delta z=Q_{1} \Delta y_{1}+Q_{2} \Delta y_{2}+\cdots+Q_{t} \Delta y_{t}$ has the smallest variance value $\sigma^{2}(\Delta z)$, which has evolved into an issue of finding the minimum value of variance $\sigma^{2}(\Delta z)$ under restricted condition $\sum_{j=1}^{t} Q_{j}=1$.

Write the equation $\Delta z=Q_{1} \Delta y_{1}+Q_{2} \Delta y_{2}+\cdots+Q_{t} \Delta y_{t}$ as:

$$
\Delta z=\left[\begin{array}{llll}
Q_{1} & Q_{2} & \cdots & Q_{t}
\end{array}\right]\left[\begin{array}{c}
\Delta y_{1} \\
\Delta y_{2} \\
\vdots \\
\Delta y_{t}
\end{array}\right]
$$

Applying the law of covariance propagation to equation (3-37) and applying formula (3-36), there is:

$$
\begin{aligned}
& \sigma^{2}(\Delta z)=\left[\begin{array}{llll}
Q_{1} & Q_{2} & \cdots & Q_{t}
\end{array}\right] \mathbf{D}(\Delta \mathbf{Y})\left[\begin{array}{c}
Q_{1} \\
Q_{2} \\
\vdots \\
Q_{t}
\end{array}\right] \\
& =\left[\begin{array}{llll}
Q_{1} & Q_{2} & \cdots & Q_{t}
\end{array}\right]\left[\mathbf{A}^{\mathrm{T}} \mathbf{P A}\right]^{-1} \mathbf{A}^{\mathrm{T}} \mathbf{P D}(\Delta \mathbf{X}) \mathbf{P A}\left[\mathbf{A}^{\mathrm{T}} \mathbf{P A}\right]^{-1}\left[\begin{array}{c}
Q_{1} \\
Q_{2} \\
\vdots \\
Q_{t}
\end{array}\right]
\end{aligned}
$$

Making $\left[\mathbf{A}^{\mathbf{T}} \mathbf{P A}\right]^{-1}\left[\begin{array}{c}Q_{1} \\ Q_{2} \\ \vdots \\ Q_{t}\end{array}\right]=\left[\begin{array}{c}1 \\ 1 \\ \vdots \\ 1\end{array}\right]$, the equation (3-38) becomes: 


$$
\sigma^{2}(\Delta z)=\left[\begin{array}{llll}
1 & 1 & \cdots & 1
\end{array}\right] \mathbf{A}^{\mathbf{T}} \mathbf{P D}(\Delta \mathbf{X}) \mathbf{P A}\left[\begin{array}{c}
1 \\
1 \\
\vdots \\
1
\end{array}\right]
$$

Besides, the restriction condition $\sum_{j=1}^{t} Q_{j}=1$ is actually:

$$
\begin{aligned}
& {\left[\begin{array}{llll}
1 & 1 & \cdots & 1
\end{array}\left[\begin{array}{c}
Q_{1} \\
Q_{2} \\
\vdots \\
Q_{t}
\end{array}\right]=\left[\begin{array}{llll}
1 & 1 & \cdots & 1
\end{array}\right]\left[\mathbf{A}^{\mathbf{T}} \mathbf{P A}\right]\left[\begin{array}{c}
1 \\
1 \\
\vdots \\
1
\end{array}\right]=1\right.} \\
& \text { That is } \quad\left(\sum_{j=1}^{t} a_{1 j}\right)^{2} P_{1}+\left(\sum_{j=1}^{t} a_{2 j}\right)^{2} P_{2}+\cdots+\left(\sum_{j=1}^{t} a_{n j}\right)^{2} P_{n}=1
\end{aligned}
$$

Similarly, according to the Lagrangian multiplier method and omitting the derivation process, the result is:

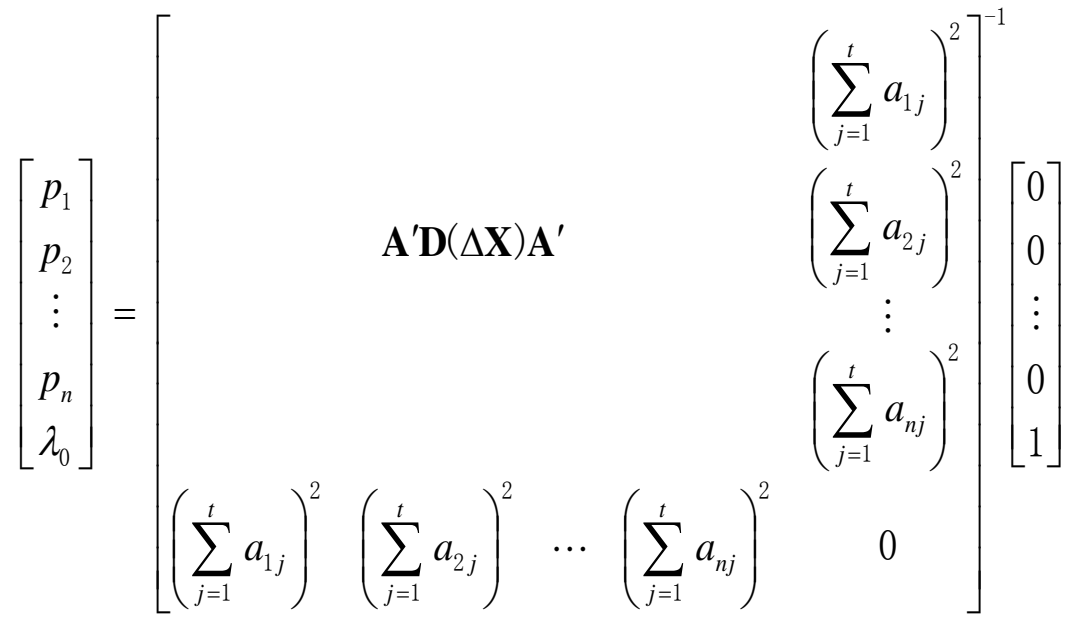

And there is

$$
\sigma^{2}(\Delta z)=-\lambda_{0}
$$

Among them, there are

$$
\mathbf{D}(\Delta \mathbf{X})=\left[\begin{array}{cccc}
\sigma_{11}^{2} & \sigma_{12} & \cdots & \sigma_{1 n} \\
\sigma_{12} & \sigma_{22}^{2} & \cdots & \sigma_{2 n} \\
\vdots & \vdots & & \vdots \\
\sigma_{1 n} & \sigma_{2 n} & \cdots & \sigma_{n n}^{2}
\end{array}\right], \mathbf{A}=\left[\begin{array}{cccc}
a_{11} & a_{12} & \cdots & a_{1 t} \\
a_{21} & a_{22} & \cdots & \sigma_{2 t} \\
\vdots & \vdots & & \vdots \\
a_{n 1} & a_{n 2} & \cdots & a_{n t}
\end{array}\right] \text { and }
$$




$$
\mathbf{A}^{\prime}=\left[\begin{array}{cccc}
\sum_{j=1}^{t} a_{1 j} & 0 & \cdots & 0 \\
0 & \sum_{j=1}^{t} a_{2 j} & \cdots & 0 \\
\vdots & \vdots & & \vdots \\
0 & 0 & \cdots & \sum_{j=1}^{t} a_{n j}
\end{array}\right]
$$

It can be seen that formula (3-26) is a special case of formula (3-41) when $t=1$, while formula (3-14) is also a special case of formula (3-27) when $a_{i} \equiv 1$.

Of course, practical applications show that formula (3-41) can indeed minimize the variance $\sigma^{2}(\Delta z)$, but it is not ideal in terms of minimizing the variance $\sigma^{2}\left(\Delta y_{j}\right)$. Therefore, this is still a question that needs further research.

\section{Conclusion}

Following the pure concept of probability theory, both the observed value and the measured value are numerical values and belong to constant, while the error and true value are unknown and belong to random variable; the variance of error is the dispersion of all possible values of the error, and is the evaluation value of the probability interval where the error exists; any error is a deviation and has its variance, so it can't be classified as systematic error or random error. Based on these concepts, and analysing the covariance propagation relationship between the observation error and the final error of measured value, it is a pure mathematical problem to give the weight assignment with the minimum variance, and the dilemma of traditional measurement theory has been solved.

In an adjustment model, the weight allocation of observations is not only determined by the covariance array of observation error, but also related to the parameters in the adjustment model, which is particularly important for a single measurand model. For the adjustment model with multiple measurands, because the errors of multiple measured values come from different propagation laws, the variances of all errors cannot be minimized at the same time, which is a question that needs further research.

\footnotetext{
References

1 JCGM 200:2012, International vocabulary of metrology — Basic and general concepts and associated terms (VIM).

2 JCGM 100:2008, Guide to the Expression of Uncertainty in Measurement(GUM).

3 JJF1001-2011, General Terms in Metrology and Their Definitions.

4 JJF1059-2012, Evaluation and Expression of Uncertainty in Measurement.

5 GB/T14911-2008, Basic Terms of Surveying and Mapping.

6 School of Geodesy and Geomatics, Wuhan University. Error Theory and Foundation of Surveying Adjustment. Wuhan University Press, 20166.

7 Xiao-ming Ye, Mo Ling, Qiang Zhou, Wei-nong Wang, Xue-bin Xiao. "The New Philosophical View about Measurement Error Theory”. Acta Metrologica Sinica, volume 36, 2015, 6, Pages: 666-670.

8 Ye Xiao-ming, Xiao Xue-bin, Shi Jun-bo, Ling Mo. The new concepts of measurement error theory [J]. Measurement, Volume 83, April 2016, Pages: 96-105. .

9 Ye Xiao-ming, Liu Hai-bo, Ling Mo, Xiao Xue-bin. The new concepts of measurement error's regularities and effect characteristics [J]. Measurement, Volume 126, October 2018, Pages: 65-71.

10 Ye Xiao-ming, Ding Shi-jun. Comparison of variance concepts interpreted by two measurement theories [J]. Journal of Nonlinear and Convex Analysis, Volume 20, Number 7, 2019, Pages: 1307-1316.

11 Shi Huisheng, Ye Xiaoming, Xing Cheng, Ding Shijun. Originand Evolution of Conceptual Differences between Two Measurement Theories [J], Fuzzy Systems and Data MiningVI, 2020.11.
} 
12 Shi Huisheng, Ye Xiaoming, Xing Cheng, Ding Shijun. A New Theoretical Interpretation of Measurement Error and Its Uncertainty [J]. Discrete Dynamics in Nature and Society, 2020. DOI: https://doi.org/10.1155/2020/3864578.

13 Ye Xiaoming. Measurement Error Theory Based on New Concepts. Hubei Science and Technology Press, 2017. 